Running head: DEPRESSIVE SYMPTOMS AND WEIGHT LOSS BEHAVIORS

\title{
Depressive Symptoms and Weight Loss Behaviors in U.S. Adults
}

Elizabeth A. Vrany, M.S. ${ }^{a}$, Misty A.W. Hawkins, Ph.D. ${ }^{\text {b }, ~ W e i ~ W u, ~ P h . D . ~}{ }^{\text {a }, ~ J e s s e ~ C . ~ S t e w a r t, ~}$ Ph.D. ${ }^{a}$

${ }^{a}$ Department of Psychology, Indiana University-Purdue University Indianapolis, Indianapolis, IN, United States

${ }^{\mathrm{b}}$ Department of Psychology, Oklahoma State University, Stillwater, OK, United States

Word Count: 3,818

Clinical Trial Registration: The data from this study do not come from a clinical trial.

Funding: This research did not receive any specific grant from funding agencies in the public, commercial, or not-for-profit sectors.

Potential Conflicts of Interest: All authors declare no conflict of interest.

Submission Declaration and Verification: This manuscript has not been published previously and is not under consideration for publication elsewhere, although some of these data were presented at the 2014 annual meeting of the American Psychosomatic Society.

Corresponding Author: Jesse C. Stewart, Ph.D., Department of Psychology, Indiana University-Purdue University Indianapolis, 402 North Blackford Street, LD 100E, Indianapolis, IN 46202. Telephone: (317) 274-6761. Fax: (317) 274-6756. Email Address: jstew@iupui.edu

This is the author's manuscript of the article published in final edited form as:

Vrany, E. A., Hawkins, M. A. W., Wu, W., \& Stewart, J. C. (2018). Depressive symptoms and weight loss behaviors in U.S. adults. Eating Behaviors, 29, 107-113. https://doi.org/10.1016/j.eatbeh.2018.03.006 


\begin{abstract}
Objective: We sought to determine whether depressive symptoms are associated with attempting to lose weight and engaging in weight loss behaviors in a large, diverse sample of adults representative of the U.S. population.

Methods: Respondents were 23,106 adults, free of cardiovascular disease and diabetes, who participated in the 2005-2014 years of the National Health and Nutrition Examination Survey (NHANES). Depressive symptoms were measured using the Patient Health Questionnaire-9 (PHQ-9), and weight loss variables were obtained from a Weight History Questionnaire.
\end{abstract} Results: PHQ-9 total was not associated with attempting to lose weight in the past year $(O R=1.03,95 \% C I=1.00-1.06, p=.074 ; n=23,106)$. Among respondents who attempted to lose weight $(n=9,582)$, PHQ-9 total was associated with a lower odds of exercising $(O R=0.84$, $95 \% C I=0.79-0.89, p<.001)$ and a greater odds of skipping meals $(O R=1.31,95 \% C I=1.22-1.41$, $p<.001)$, eating diet foods/products $(O R=1.16,95 \% C I=1.08-1.24, p<.001)$, eating less food $(O R=1.09,95 \% C I=1.04-1.15, p<.001)$, taking non-prescription supplements $(O R=1.31$, $95 \% C I=1.23-1.41, p<.001)$, taking prescription diet pills $(O R=1.28,95 \% C I=1.10-1.49, p=.001)$, and taking laxatives/vomiting $(O R=1.55,95 \% C I=1.28-1.88, p<.001)$.

Conclusions: Although depressive symptoms were not associated with attempting to lose weight in the past year, adults who attempted to lose weight tended to employ potentially ineffective/unhealthy weight loss behaviors and avoid effective behaviors. This pattern of behaviors may be another mechanism that explains the excess risk of obesity in depressed adults and may be a modifiable target for future interventions. Given the cross-sectional nature of this study, reverse causality is a possibility. Future studies should investigate the prospective associations between depressive symptoms and weight loss behaviors. 
Keywords: depression; weight loss behaviors; diet; physical activity; obesity

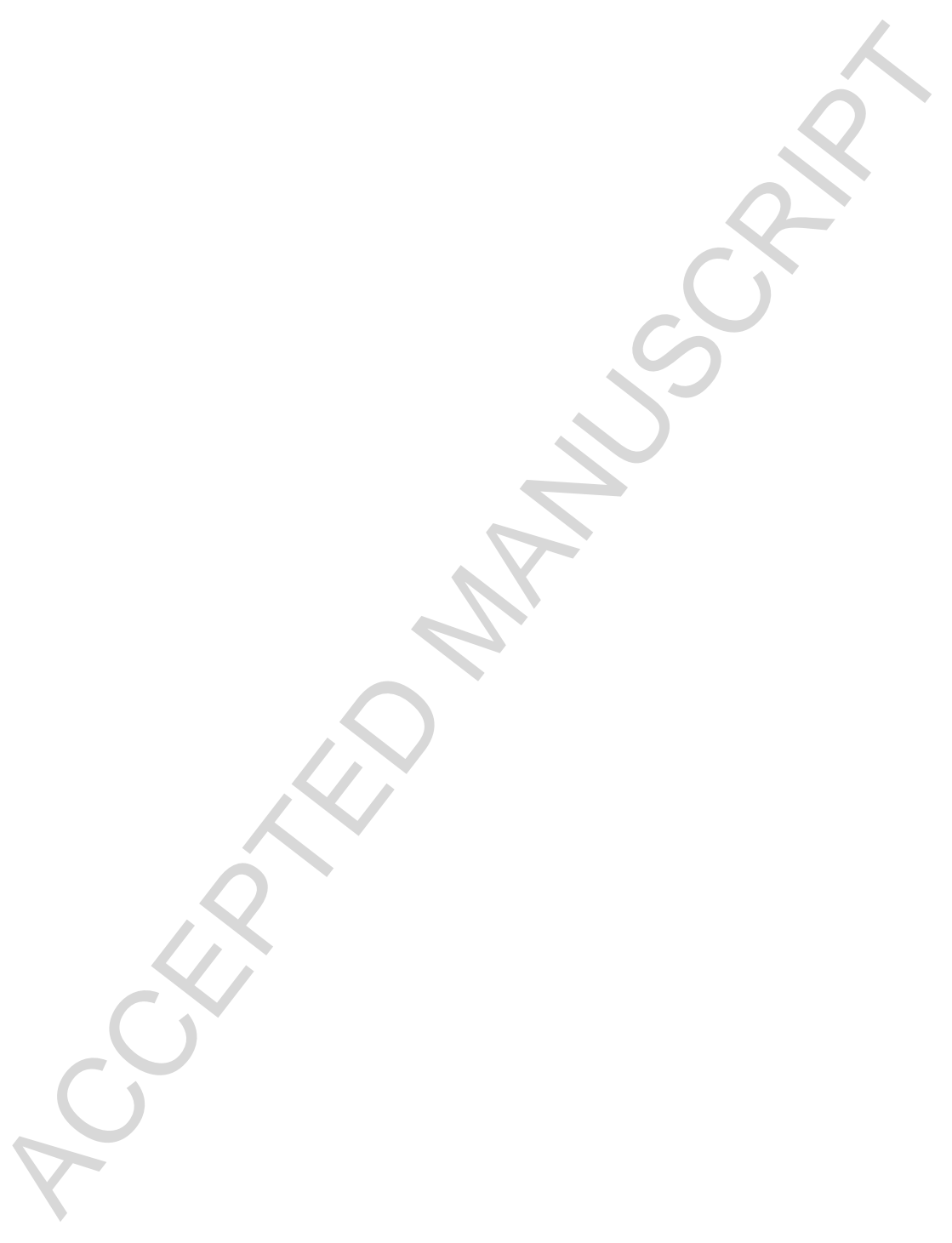




\section{Introduction}

A meta-analysis of prospective studies indicates that depression is a risk factor for obesity. ${ }^{1}$ The depression-to-obesity relationship has been observed among various age groups, ${ }^{1}$ in both men and women, ${ }^{1,2}$ and across multiple racial and ethnic groups. ${ }^{3,4}$ Although the exact pathways have yet to be elucidated, candidate mechanisms have been proposed to explain how depression may promote obesity. Candidate biological mechanisms include increased systemic inflammation, ${ }^{5}$ hypothalamic-pituitary-adrenal axis dysregulation, ${ }^{6}$ and metabolic dysfunction, ${ }^{7}$ and candidate behavioral mechanisms include emotional eating, ${ }^{8}$ disordered eating, ${ }^{9}$ consumption of calorie dense foods, ${ }^{10}$ physical inactivity, ${ }^{11}$ and psychotropic medication use. ${ }^{12}$

Another candidate behavioral mechanism that has received limited attention is engaging in ineffective weight loss behaviors. While evidence indicates that depressed people are less likely to be adherent to medical recommendations pertaining to weight $\operatorname{loss}^{13}$ and depression is a risk factor for the development of eating disorder pathology, ${ }^{14,15}$ few studies have examined whether depression is associated with the likelihood of using effective or ineffective weight loss behaviors. Behaviors known to effectively promote weight loss include reducing calorie or fat intake, eating less food, reducing sweet consumption, increasing physical activity, and selfmonitoring weight and food intake. ${ }^{16-18}$ In contrast, ineffective or unhealthy weight loss behaviors include skipping meals, fasting, consuming certain "fad diet" food products, taking diet pills, using laxatives, purging, and smoking. ${ }^{16,18,19}$

Elevated depressive symptoms have been inversely associated with physical activity, an effective weight loss behavior. ${ }^{20,21}$ Moreover, elevated depressive symptoms have been positively associated with engaging in some ineffective weight loss behaviors. ${ }^{19,22,23}$ For instance, in a sample of 404 undergraduate students with overweight or obesity, elevated 
depressive symptoms were related to engaging in one or more unhealthy weight loss behaviors. ${ }^{23}$ In another study involving 106 children and adolescents, depressive symptoms were found to partially mediate the positive relationship between perception of overweight and number of unhealthy weight loss behaviors. ${ }^{19}$ Associations between depressive symptoms and weight loss behaviors vary by gender. In a sample of 198 undergraduate students, women with higher depressive symptoms used fewer healthy weight loss behaviors (e.g., eating fruits and vegetables) and more unhealthy weight loss behaviors (e.g., skipping meals) than nondepressed women. ${ }^{22}$ Depressive symptoms were not related to use of healthy or unhealthy weight loss behaviors in men.

To date, no study has examined the associations of depressive symptoms with effective and ineffective weight loss behaviors in a sample of adults representative of the U.S. population. Accordingly, the present study's objectives were: (1) to determine whether depressive symptom severity is associated with attempting to lose weight in the past year in a large, diverse, and representative sample of U.S. adults; (2) to determine whether depressive symptom severity is related to specific weight loss behaviors among adults who attempted to lose weight in the past year; and (3) to explore gender and race/ethnicity as moderators of depressive symptoms-weight loss behaviors relationships. It is important to examine these moderators because (a) the depression-obesity association may vary by gender and race/ethnicity (i.e., the relationship may be stronger among women ${ }^{24}$ and non-Hispanic whites ${ }^{24,25}$ ), and (b) although rates of obesity are highest among African American women, ${ }^{25}$ concern regarding weight status is lowest in this group, ${ }^{26-28}$ potentially contributing to lesser engagement in weight management behaviors. These aims were achieved by examining data collected as part of the National Health and Nutrition Examination Survey (NHANES) 2005-2014, which contains measures of depressive symptoms 
and weight loss behaviors. Identifying the weight loss behaviors of depressed adults will inform mechanistic models of the depression-to-future-obesity relationship and will help to identify modifiable targets for interventions designed to prevent or manage depression's contribution to obesity.

\section{Materials and Methods}

This study examined cross-sectional data from the 2005-2014 years of NHANES, a survey conducted by the National Center for Health Statistics of the Centers for Disease Control and Prevention examining the health and nutritional status of the U.S. population. Data were collected from a sample representative of the civilian, non-institutionalized U.S. population.

Those who were selected to participate were first interviewed in their homes by trained NHANES personnel. One to two weeks later, respondents traveled to a Mobile Examination Center (MEC), where they completed additional interviews and underwent laboratory and examination assessments. Our archival study was approved by the institutional review board at Indiana University-Purdue University Indianapolis (IUPUI). Detailed information regarding the study design is provided elsewhere (www.cdc.gov/nchs/nhanes.htm).

From the total sample of 50,965 respondents for the 2005-2014 survey years, we selected all respondents 18 years and older $(n=30,295)$. To obtain a generally healthy sample and minimize the influence of potential confounders, we applied our exclusion criteria. First, we excluded respondents with cardiovascular disease or diabetes $(n=5,543)$ to ensure that observed relationships between depression and weight loss behaviors were not driven by these medical conditions, as both conditions have independent relationships with depression ${ }^{29,30}$ and involve specific dietary or physical activity recommendations. ${ }^{31,32}$ Both cardiovascular disease and diabetes were measured via a self-report item asking, "Has a doctor or other health professional 
ever told you that you had [medical condition]?" Second, we excluded those with a body mass index $(\mathrm{BMI})$ potentially indicative of anorexia nervosa $\left(<17.5 \mathrm{~kg} / \mathrm{m}^{2}\right)(n=202)$. Including those with anorexia would artificially inflate the association between depression and unhealthy weight loss behaviors, as depression is common in adults with anorexia and anorexia is characterized by the use of unhealthy weight loss behaviors. ${ }^{33}$ Third, we excluded those who were pregnant at the time of assessment due to the influence of pregnancy on depression, body weight, and weightrelated behaviors $(n=598) .{ }^{34}$ Fourth, we excluded those who did not attend the MEC assessment ( $n=846)$ because the MEC sample weight variable was used in the analyses. Applying these exclusions yielded Sample \#1 of 23,106 adults. We further selected respondents who answered "yes" to the tried to lose weight item to establish Sample \#2 of 9,582 respondents with a pastyear weight loss attempt. Sample characteristics are shown in Table 1.

\subsection{Depressive Symptom Severity}

The Patient Health Questionnaire-9 (PHQ-9) ${ }^{35}$ was administered by NHANES personnel during the MEC interviews. Respondents indicated the frequency, on a 0-3 scale, with which they experienced nine symptoms of depression over the past two weeks. Total scores range from 0 to 27 , and scores $\geq 10$ represent clinically significant depressive symptoms. ${ }^{35}$ The PHQ-9 has high internal consistency and good sensitivity and specificity for identifying major depressive disorder in community samples. ${ }^{35}$ We calculated the PHQ-9 total by summing the nine items. We additionally computed a dichotomous PHQ-9 variable to identify those with (PHQ-9 $\geq 10$ ) or without (PHQ-9<10) clinically significant depressive symptoms.

\subsection{Past-Year Weight Loss Attempt and Behaviors}

To create variables for past-year weight loss attempt and behaviors, we used items from the Weight History Questionnaire. During the home interview, NHANES personnel asked 
respondents to self-report their current weight and their weight one year ago. Respondents who reported losing $\geq 10$ pounds in the past year were asked, "Was the change in your current weight and your weight a year ago because you tried to lose weight?" Respondents who reported losing $<10$ pounds in the past year were asked, "During the past 12 months, have you tried to lose weight?" We coded those who answered "yes" to either of these questions - and thus reported trying to lose weight in the past year, regardless of weight loss success - as "yes" on our pastyear weight loss attempt variable. All other respondents were coded as "no."

Respondents with a past-year weight loss attempt were asked two follow-up questions. First, they were asked "How did you try to lose weight?" in the past year and were presented with a card that listed 18 weight loss behaviors. For all behaviors on the card selected by the respondents, we coded them as "yes" on our corresponding past-year weight loss behavior variables. For all unselected behaviors, respondents were coded as "no." Second, respondents were asked "In the past 12 months, did you seek help from a personal trainer, dietitian, nutritionist, doctor, or other health professional?" Those who responded "yes" were coded as seeking help from a health professional in the past year, and all others were coded as "no." From these two questions, we created 19 past-year weight loss behavior variables (see Figure 1). To streamline the presentation of results, we organized the behaviors into categories: dietary, physical activity, medication, help seeking, and miscellaneous behaviors.

\subsection{Covariates}

Models included the following covariates: age (years), gender $(0=$ male, $1=$ female $)$, three dummy-coded variables for race/ethnicity, education, and BMI $\left(\mathrm{kg} / \mathrm{m}^{2}\right)$. Data for the demographic covariates were obtained during the home interviews. The primary NHANES race/ethnicity variable has five levels: non-Hispanic White, non-Hispanic Black, Mexican 
American, Other Hispanic, and Other. Because the counts for the Other and Other Hispanic groups were low we combined them into one heterogeneous Other group. We computed three dummy-coded variables comparing the non-Hispanic Black (RE1), Mexican American (RE2) and the Other group (RE3) groups to the non-Hispanic White reference group. Respondents reported their highest level of education, which was coded in the following categories: less than 9th grade, 9-12th grade with no diploma, high school diploma or GED, some college or associates degree, and college graduate or above. BMI was calculated using measurements of height and weight obtained during the MEC examination.

\subsection{Data Analyses}

We used multiple imputation to handle missing data for PHQ-9 total (Sample \#1 $n=2,468$; Sample \#2 n=880), BMI (Sample \#1 n=266; Sample \#2 n=87), education (Sample \#1 $n=27$; Sample \#2 n=3), and the tried to lose weight item (Sample \#1 n=50). Specifically, we used SAS 9.4 to obtain 20 imputed datasets with 200 burn-in iterations using the PROC MI Fully Conditional Specification method. ${ }^{36}$ In the imputation model, we included variables that predicted the missingness of the incomplete variables as well as interaction terms to preserve potential nonlinear relationships. To account for stratum and cluster effects, we included dummy variables for stratum and cluster effects in the imputation. ${ }^{37}$ Following imputation, a series of logistic regression models (described below) were fit to each of the imputed datasets using SAS PROC SURVEYLOGISTIC. The estimates for the multiply imputed datasets were then combined using SAS PROC MIANALYZE. ${ }^{36}$

To determine whether depressive symptom severity is associated with attempting to lose weight in the past year, we fit a logistic regression model for Sample \#1. PHQ-9 total (z-scored) was entered as the predictor variable, and past-year weight loss attempt was entered as the 
criterion variable. Covariates were age, gender, three dummy-coded race/ethnicity variables, education, and BMI. To illustrate clinical relevance, we re-ran this model replacing the continuous PHQ-9 total variable with the dichotomous PHQ-9 variable.

To determine whether depressive symptom severity is related to specific weight loss behaviors among adults who attempted to lose weight in the past year, we ran a series of logistic regression models for Sample \#2. Separate models were constructed for each of the 19 weight loss behavior variables. For each model, the PHQ-9 total (z-scored) was entered as the predictor variable, and the selected weight loss behavior variable was entered as the criterion variable. Covariates in all models were age, gender, three dummy-coded race/ethnicity variables, education, and BMI. We adjusted for multiple comparisons using the Holm-Bonferroni approach, which reduces risk of type I error with less risk of over-correction than the Bonferroni approach. ${ }^{38}$ To illustrate clinical relevance, we re-ran all models for significant depressive symptom-weight loss behavior relationships replacing the continuous PHQ-9 total variable with the dichotomous PHQ-9 variable.

To explore gender and race/ethnicity as moderators of the depressive symptom severityweight loss behaviors relationships, we computed two cross-product interaction terms by multiplying PHQ-9 total ( $z$-scored) by gender (female vs. male) and race/ethnicity (white vs. non-white). To test gender as a moderator, the PHQ-9 $\mathrm{x}$ gender interaction term was entered into a series of logistic regression models with each of the 19 weight loss behaviors entered separately as the criterion variable (Sample \#2). Covariates were age, gender, race/ethnicity, education, BMI, and PHQ-9 total. A parallel set of analyses was conducted for the PHQ-9 $\mathrm{x}$ race/ethnicity interaction term. In the presence of significant interactions, we reran the logistic regression models after stratifying by gender or race/ethnicity. 
Note that estimates from the logistic regression analyses were weighted using NHANES sampling design variables - i.e., strata, primary sampling using, and MEC sample weights which account for survey design factors including oversampling, survey non-response, and poststratification (for further details, see www.cdc.gov/nchs/tutorials/nhanes/SurveyDesign/Weighting/intro.htm). Analyses utilizing NHANES sampling design variables provide estimates representative of the U.S. civilian noninstitutionalized population.

\section{Results}

\subsection{Depressive Symptoms and Weight Loss Attempts (Sample \#1)}

As is shown in Table 1, the mean age of Sample \#1 was 44 years, and 51\% were female and $44 \%$ were non-white. The mean BMI fell in the overweight category. The mean PHQ-9 fell in the minimal depression category, although $8 \%$ exhibited clinically significant depressive symptoms. A logistic regression model revealed that depressive symptoms were not associated with attempting to lose weight in the past year, although the $p$-value fell just short of significance $(O R=1.03,95 \% C I=1.00-1.06, p=.074)$. Another logistic regression model, entering the dichotomous PHQ-9 variable as the predictor variable, indicated that clinically significant depressive symptoms were not associated with odds of attempting to lose weight in the past year $(p=.958)$.

\subsection{Depressive Symptoms and Weight Loss Behaviors (Sample \#2)}

Table 1 shows that the characteristics of Sample \#2 were similar to those of Sample \#1. Notably, there were more women in Sample \#2, and the mean BMI fell in the obesity category. We ran separate logistic regression models, adjusted for demographic factors and BMI, for each 
weight loss behavior. Original $p$-values are reported, but only $p$-values $<.05$ after HolmBonferroni adjustment are identified as significant.

Depressive symptom severity was related to seven of the 19 weight loss behaviors after applying the Holm-Bonferroni adjustment (see Figure 1). For the 12 dietary behaviors, a 1-SD increase (4.1 points) in PHQ-9 total was associated with a $31 \%$ greater odds of skipping meals, a $16 \%$ greater odds of eating diet foods or products, and a $9 \%$ greater odds of eating less food. PHQ-9 total was not related to the remaining nine dietary behaviors. For the one physical activity behavior, a 1-SD increase in PHQ-9 total was associated with a $16 \%$ lower odds of exercising. For the two medication behaviors, a 1-SD increase in PHQ-9 total was associated with a $31 \%$ greater odds of taking non-prescription supplements and a $28 \%$ greater odds of taking prescription diet pills. PHQ-9 total was not related to the two help seeking behaviors. For the two miscellaneous behaviors, a 1-SD increase in PHQ-9 total was associated with a 55\% greater odds of taking laxatives or vomiting but not starting or resuming smoking to lose weight.

To illustrate clinical relevance of these findings, we re-ran the logistic regression models with the dichotomous PHQ-9 variable predicting the seven weight loss behaviors that were significantly associated with the continuous PHQ-9 variable (see Table 2). Compared to their nondepressed counterparts (PHQ-9<10), respondents with clinically significant depressive symptoms (PHQ-9 $\geq 10$ ) had an $88 \%$ greater odds of skipping meals, a $40 \%$ greater odds of eating diet foods or products, a $46 \%$ greater odds of eating less food, a 35\% lower odds of exercising, a $74 \%$ greater odds of taking non-prescription supplements, and a 334\% greater odds of taking laxatives or vomiting. Although the continuous PHQ-9 score variable was positively associated with taking prescription diet pills, having clinically significant depressive symptoms was not significantly associated with odds of taking prescription diet pills. However, the magnitude of 
the odds ratio suggests that low statistical power may be partially responsible for this nonsignificant result.

\subsection{Exploratory Analyses: Gender and Race/Ethnicity as Moderators of Depressive}

\section{Symptoms-Weight Loss Behavior Relationships (Sample \#2)}

Moderation by gender was detected for two (13\%) of the 19 weight loss behaviors - i.e., ate diet foods or products and joined a weight loss program (see Supplemental Table 1).

Stratified analyses indicated that greater depressive symptom severity is associated with a greater odds of eating diet foods and products in men than in women. Although a significant interaction was detected for joined a weight loss program, stratified analyses revealed a trend for greater depressive symptoms being associated with greater odds of this weight loss behavior in men and no relationship in women.

Moderation by race/ethnicity was detected for three (16\%) of the 19 weight loss behaviors - i.e., took non-prescription supplements, took laxatives or vomited, and started to smoke or resumed smoking (see Supplemental Table 2). Stratified analyses demonstrated that greater depressive symptom severity was associated with (1) a greater odds of taking nonprescription supplements in white than in non-white respondents; (2) a greater odds of taking laxatives or vomiting in white than in non-white respondents; and (3) a lower odds of smoking to lose weight in white than in non-white respondents.

\section{Discussion}

In a large, diverse sample of adults representative of the U.S. population free of cardiovascular disease and diabetes, we found that depressive symptom severity was not associated the odds of attempting to lose weight in the past year. However, among adults who attempted to lose weight in the past year, those with higher depressive symptoms tended to 
employ weight loss behaviors likely to be ineffective or unhealthy - namely, skipping meals, eating diet foods or products, taking non-prescription supplements, taking prescription diet pills, and taking laxatives or vomiting. Moreover, adults with higher depressive symptoms tended not to use exercise as a weight loss behavior, which has been shown to be effective in weight maintenance and contribute to weight loss at high levels. ${ }^{17}$ Interestingly, depressive symptom severity was positively related to one potentially effective weight-loss strategy - eating less food. The observed relationships are not trivial in size and are potentially clinically relevant, as results largely replicated when examining associations between clinically elevated depressive symptoms and weight loss behaviors. Exploratory analyses examining moderators of the depressive symptoms-weight loss behavior associations suggest differences in a few of these relationships by gender and race/ethnicity. Collectively, the pattern of results suggests that, when adults with elevated depressive symptoms attempt to lose weight, they utilize different - and likely less effective - behavioral strategies than adults with no or minimal depressive symptoms. Our findings raise the possibility that engaging in potentially ineffective or unhealthy weight loss behaviors and failing to engage in effective weight loss behaviors may be two additional factors that contribute to the elevated risk of obesity of depressed people. However, the possibility of reverse causality or the presence of confounding variables should not be overlooked.

To our knowledge, this study is the first to examine associations between depressive symptom severity and multiple weight loss behaviors in a sample of adults representative of the U.S. population, which is a significant extension of past findings. Our results are consistent with previous studies in smaller samples of children, adolescents, and undergraduate students, which indicate that elevated depressive symptoms are associated with more frequent use of unhealthy weight loss behaviors. ${ }^{19,22,23}$ Our results are in line with studies that have shown that elevated 
depressive symptoms are inversely associated with engaging in physical activity ${ }^{20,21}$ and predict greater eating disorder pathology. ${ }^{14,15}$

There are several potential explanations for why elevated depressive symptoms were associated with an increased odds of engaging in potentially ineffective or unhealthy weight loss behaviors and a decreased odds of engaging in effective weight loss behaviors. First, specific depressive symptoms may play a role in the selection of weight loss behaviors. For instance, anhedonia and fatigue could promote the selection of weight loss behaviors that require less physical effort (e.g., using diet pills and eating diet foods or products) and the avoidance of weight loss behaviors that require more physical effort (e.g., exercise). Additionally, those experiencing the depressive symptom of appetite disturbance may be more likely to skip meals due to decreased hunger. Second, adults with elevated depressive symptoms may use weight loss behaviors that require less planning and self-efficacy (e.g., skipping meals or vomiting) and avoid behaviors that require more planning and self-efficacy (e.g., exercising). Depression has been linked with deficits in executive functioning ${ }^{39}$ and lower self-efficacy. ${ }^{40-43}$ Third, because depressed adults have higher rates of healthcare utilization, ${ }^{44}$ those with elevated depressive symptoms may be more likely to seek prescription diet medications. Future studies are needed to evaluate these and other potential mechanism underlying the observed relationships.

Our study has limitations that are worth noting. First, due to the cross-sectional NHANES design, we are unable to determine the directionality of the observed associations. We discuss our findings in the direction of depressive symptoms leading to the use of certain weight loss behaviors; however, reverse causality is a possibility. For instance, engagement in weight loss behaviors could lead to elevated depressive symptoms through mechanisms such as low selfesteem or low satisfaction with weight loss progress, particularly when using ineffective 
behaviors. Additionally, certain ineffective strategies (e.g., skipping meals, eating less food, and using diet pills) could contribute to the development of specific depressive symptoms (e.g., fatigue or difficulties with concentration, sleep, and appetite). Moreover, physical activity, even at low levels, can buffer against the development of depression. ${ }^{45}$ Future prospective studies are needed to examine directionality. Second, we were unable to control for some potentially confounding variables, including eating disorders and weight and shape concerns, as these variables were not assessed by NHANES. Third, weight loss behaviors were measured via selfreport, which is subject to social desirability and may result in the under-reporting of negatively perceived behaviors (e.g., took laxatives/vomited) and over-reporting of positively perceived behaviors (e.g., ate less food). Fourth, because the weight loss behaviors were measured as yes/no responses to a list of behaviors, we could not examine relationships between depressive symptoms and the frequency, duration, or intensity of the weight loss behaviors. Despite these limitations, our study also has several important strengths, including a large, diverse sample representative of the U.S. population, a strong assessment of depressive symptoms, and the simultaneous examination of multiple weight loss behaviors.

\section{Conclusions}

The present results indicate that, although depressive symptoms were not associated with attempting to lose weight in the past year, adults who attempted to lose weight tended to employ potentially ineffective or unhealthy weight loss behaviors and avoid effective behaviors. Because this pattern of behaviors may be yet another mechanism that explains the excess risk of obesity in depressed adults, our findings could inform mechanistic models of the depression-to-futureobesity relationship. Given that this study utilized cross-sectional data and reverse causality is a possibility, future prospective studies are needed to examine directionality. In addition, our 
findings could also help to identify modifiable targets for interventions aimed at preventing or managing depression's contribution to obesity in U.S. adults. Recent clinical trials demonstrate that simultaneous and sequential approaches to intervening on depression and weight loss in women with obesity result in reductions in body weight and depression. ${ }^{46-48}$ If findings from the present study are replicated using prospective data, future interventions could simultaneously target depressive symptoms and obesity-related behaviors, with specific treatment modules focusing on bolstering the use of effective behaviors and halting the use of ineffective behaviors. 


\section{References}

1. Luppino FS, de Wit LM, Bouvy PF, et al. Overweight, obesity, and depression: A systematic review and meta-analysis of longitudinal studies. Arch Gen Psychiatry. 2010;67(3):220-229.

2. Roberts RE, Deleger S, Strawbridge WJ, Kaplan GA. Prospective association between obesity and depression: evidence from the Alameda County Study. Int J Obes Relat Metab Disord. 2003;27(4):514-521.

3. Franko DL, Striegel-Moore RH, Thompson D, Schreiber GB, Daniels SR. Does adolescent depression predict obesity in black and white young adult women? Psychol Med. 2005;35(10):1505-1513.

4. Merten MJ, Wickrama K, Williams AL. Adolescent obesity and young adult psychosocial outcomes: Gender and racial differences. J Youth Adolesc. 2008;37(9):1111-1122.

5. Miller GE, Freedland KE, Carney RM, Stetler CA, Banks WA. Pathways linking depression, adiposity, and inflammatory markers in healthy young adults. Brain Behav Immun. 2003;17(4):276-285.

6. Bornstein SR, Schuppenies A, Wong ML, Licinio J. Approaching the shared biology of obesity and depression: the stress axis as the locus of gene-environment interactions. Mol Psychiatry. 2006;11(10):892-902.

7. Skilton MR, Moulin P, Terra JL, Bonnet F. Associations between anxiety, depression, and the metabolic syndrome. Biol Psychiatry. 2007;62(11):1251-1257.

8. Konttinen H, Silventoinen K, Sarlio-Lahteenkorva S, Mannisto S, Haukkala A. Emotional eating and physical activity self-efficacy as pathways in the association 
between depressive symptoms and adiposity indicators. Am J Clin Nutr. 2010;92(5):1031-1039.

9. Abrams KK, Allen LR, Gray JJ. Disordered eating attitudes and behaviors, psychological adjustment, and ethnic identity: a comparison of black and white female college students. Int J Eat Disord. 1993;14(1):49-57.

10. Tomiyama AJ, Dallman MF, Epel ES. Comfort food is comforting to those most stressed: evidence of the chronic stress response network in high stress women. Psychoneuroendocrinology. 2011;36(10):1513-1519.

11. Must A, Tybor DJ. Physical activity and sedentary behavior: a review of longitudinal studies of weight and adiposity in youth. Int J Obes (Lond). 2005;29 Suppl 2:S84-96.

12. Smits JA, Rosenfield D, Mather AA, Tart CD, Henriksen C, Sareen J. Psychotropic medication use mediates the relationship between mood and anxiety disorders and obesity: findings from a nationally representative sample. J Psychiatr Res. 2010;44(15):1010-1016.

13. Berntson J, Stewart KR, Vrany E, Khambaty T, Stewart JC. Depressive symptoms and self-reported adherence to medical recommendations to prevent cardiovascular disease: NHANES 2005-2010. Soc Sci Med. 2015;138:74-81.

14. Puccio F, Fuller-Tyszkiewicz M, Ong D, Krug I. A systematic review and meta-analysis on the longitudinal relationship between eating pathology and depression. Int J Eat Disord. 2016;49(5):439-454.

15. Stice E. Risk and maintenance factors for eating pathology: a meta-analytic review. Psychol Bull. 2002;128(5):825-848. 
16. Nicklas JM, Huskey KW, Davis RB, Wee CC. Successful weight loss among obese U.S. adults. Am J Prev Med. 2012;42(5):481-485.

17. Swift DL, Johannsen NM, Lavie CJ, Earnest CP, Church TS. The role of exercise and physical activity in weight loss and maintenance. Prog Cardiovasc Dis. 2014;56(4):441447.

18. French SA, Perry CL, Leon GR, Fulkerson JA. Dieting behaviors and weight change history in female adolescents. Health Psychol. 1995;14(6):548-555.

19. Armstrong B, Westen SC, Janicke DM. The role of overweight perception and depressive symptoms in child and adolescent unhealthy weight control behaviors: a mediation model. J Pediatr Psychol. 2014;39(3):340-348.

20. Roshanaei-Moghaddam B, Katon WJ, Russo J. The longitudinal effects of depression on physical activity. Gen Hosp Psychiatry. 2009;31(4):306-315.

21. Vallance JK, Winkler EA, Gardiner PA, Healy GN, Lynch BM, Owen N. Associations of objectively-assessed physical activity and sedentary time with depression: NHANES (2005-2006). Prev Med. 2011;53(4-5):284-288.

22. Gillen MM, Markey CN, Markey PM. An examination of dieting behaviors among adults: links with depression. Eat Behav. 2012;13(2):88-93.

23. Davila EP, Kolodziejczyk JK, Norman GJ, et al. Relationships between depression, gender, and unhealthy weight loss practices among overweight or obese college students. Eat Behav. 2014;15(2):271-274.

24. de Wit L, Luppino F, van Straten A, Penninx B, Zitman F, Cuijpers P. Depression and obesity: a meta-analysis of community-based studies. Psychiatry Res. 2010;178(2):230235. 
25. Ogden CL, Carroll MD, Kit BK, Flegal KM. Prevalence of obesity among adults: United States, 2011-2012. NCHS Data Brief. 2013(131):1-8.

26. Neumark-Sztainer D, Croll J, Story M, Hannan PJ, French SA, Perry C. Ethnic/racial differences in weight-related concerns and behaviors among adolescent girls and boys: findings from Project EAT. J Psychosom Res. 2002;53(5):963-974.

27. Paeratakul S, White MA, Williamson DA, Ryan DH, Bray GA. Sex, race/ethnicity, socioeconomic status, and BMI in relation to self-perception of overweight. Obes Res. 2002;10(5):345-350.

28. White MA, Kohlmaier JR, Varnado-Sullivan P, Williamson DA. Racial/ethnic differences in weight concerns: protective and risk factors for the development of eating disorders and obesity among adolescent females. Eat Weight Disord. 2003;8(1):20-25.

29. Renn BN, Feliciano L, Segal DL. The bidirectional relationship of depression and diabetes: a systematic review. Clin Psychol Rev. 2011;31(8):1239-1246.

30. Thombs BD, Bass EB, Ford DE, et al. Prevalence of depression in survivors of acute myocardial infarction. J Gen Intern Med. 2006;21(1):30-38.

31. Pearson TA, Palaniappan LP, Artinian NT, et al. American Heart Association Guide for Improving Cardiovascular Health at the Community Level, 2013 update: a scientific statement for public health practitioners, healthcare providers, and health policy makers. Circulation. 2013;127(16):1730-1753.

32. American Diabetes Association. Foundations of care and comprehensive medical evaluation. Sec. 3. In Standards of Medical Care in Diabetes. Diabetes Care. 2016;39(Suppl. 1):S23-S35.

33. Fairburn CG, Harrison PJ. Eating disorders. Lancet. 2003;361(9355):407-416. 
34. Vesga-Lopez O, Blanco C, Keyes K, Olfson M, Grant BF, Hasin DS. Psychiatric disorders in pregnant and postpartum women in the United States. Arch Gen Psychiatry. 2008;65(7):805-815.

35. Kroenke K, Spitzer RL. The PHQ-9: a new depression diagnostic and severity measure. Psychiatric Annals. 2002;32:509-515.

36. Berglund PA. Multiple imputation using the fully conditional specification method: A comparison of SAS®, Stata, IVEware, and R. Proceedings of the SAS Global Forum 2015 Conference. Cary, NC: SAS Institute Inc. 2015; Available from: http://support.sas.com/resources/papers/proceedings15/2081-2015.pdf Accessed on 10 February 2018.

37. Reiter JP, Raghunathan TE, Kinney SK. The importance of modeling the sampling design in multiple imputations for missing data. Survey Methodology. 2006;32(2):143150.

38. Holm S. A simple sequentially rejective multiple test procedure. Scand Stat Theory Appl. 1979;6:65-70.

39. Snyder HR. Major depressive disorder is associated with broad impairments on neuropsychological measures of executive function: a meta-analysis and review. Psychol Bull. 2013;139(1):81-132.

40. Maddux JE, Meier LJ. Self-Efficacy and Depression. In: Maddux JE, ed. Self-Efficacy, Adaptation, and Adjustment. New York: Plenum Press; 1995.

41. Lin K, Park C, Li M, et al. Effects of depression, diabetes distress, diabetes self-efficacy, and diabetes self-management on glycemic control among Chinese population with type 2 diabetes mellitus. Diabetes Res Clin Pract. 2017;131:179-186. 
42. Devarajooh C, Chinna K. Depression, distress and self-efficacy: The impact on diabetes self-care practices. PLoS One. 2017;12(3):e0175096.

43. Mystakidou K, Parpa E, Tsilika E, et al. Self-efficacy, depression, and physical distress in males and females with cancer. Am J Hosp Palliat Care. 2010;27(8):518-525.

44. Koopmans GT, Donker MC, Rutten FH. Common mental disorders and use of general health services: a review of the literature on population-based studies. Acta Psychiatr Scand. 2005;111(5):341-350.

45. Mammen G, Faulkner G. Physical activity and the prevention of depression: a systematic review of prospective studies. Am J Prev Med. 2013;45(5):649-657.

46. Faulconbridge LF, Wadden TA, Berkowitz RI, Pulcini ME, Treadwell T. Treatment of comorbid obesity and major depressive disorder: A prospective pilot study for their combined treatment. J Obes. 2011;2011:870385.

47. Linde JA, Simon GE, Ludman EJ, et al. A randomized controlled trial of behavioral weight loss treatment versus combined weight loss/depression treatment among women with comorbid obesity and depression. Ann Behav Med. 2011;41(1):119-130.

48. Pagoto S, Schneider KL, Whited MC, et al. Randomized controlled trial of behavioral treatment for comorbid obesity and depression in women: The Be Active Trial. Int $J$ Obes (Lond). 2013;37(11):1427-1434. 
Entire Sample

$n=\mathbf{2 3 , 1 0 6}$

$44.3(18.0)$

Female, \%

Race/Ethnicity

Non-Hispanic White, \%

Non-Hispanic Black, \%

Mexican American, \%

Other, \%

Less than 9 th Grade, $\%^{\mathrm{a}}$

Body Mass Index, $\mathrm{kg} / \mathrm{m}^{2}, M(S D)^{\mathrm{b}}$

PHQ-9 Total (range: 0-27), $M(S D)^{\mathrm{c}}$

PHQ-9 Total $\geq 10, \%{ }^{\mathrm{c}}$
Sample \#1:

50.9

Sample \#2:

Subsample with a

Past-Year Weight

Loss Attempt

$\boldsymbol{n}=\mathbf{9 , 5 8 2}$

$42.9(16.5)$

61.2

43.6

44.3

21.2

21.1

16.6

16.7

18.6

17.9

9.0

5.9

$28.4(6.6)$

$2.9(4.0)$

$30.9(6.8)$

$3.1(4.1)$

7.6

Note. PHQ-9=Patient Health Questionnaire-9.

${ }^{a}$ Based on a reduced sample of 23,079 respondents in Sample \#1 and 9,579 respondents in Sample \#2 with complete data for education.

${ }^{\mathrm{b}}$ Based on a reduced sample of 22,840 respondents in Sample \#1 and 9,495 respondents Sample \#2 with complete data for body mass index.

${ }^{\mathrm{c}}$ Based on a reduced sample of 20,638 respondents in Sample \#1 and 8,702 respondents in Sample \#2 with complete data for the PHQ-9. 
Table 2. Results of Logistic Regression Models Examining the Relationship Between Clinically Significant Depressive Symptoms $(P H Q-9 \geq 10)$ and Weight Loss Behaviors

\section{Dietary Behaviors}

Skipped meals

Ate diet foods or products

Ate less food

Physical Activity Behaviors

Exercised

\section{Medication Behaviors}

Took non-prescription

supplements

Took prescription diet pills
0.65

0.54

0.77

$<.001 *$

1.88

1.54

1.03

2.30

1.90

1.74

$<.001 *$

$0.031^{*}$

$<.001 *$

1.22

1.46

$<.001^{*}$

$p$-value

$+$

Miscellaneous Behaviors

Took laxatives or vomited

3.34

1.53

7.29

$0.002 *$

Note: $n=9,582$. Examined only those weight loss behaviors found to be significantly associated with the continuous PHQ-9 score. All models are adjusted for age, gender, race/ethnicity, education level, body mass index, and NHANES sampling design. PHQ9=Patient Health Questionnaire-9. OR=odds ratio. $\mathrm{CI}=$ confidence interval. 


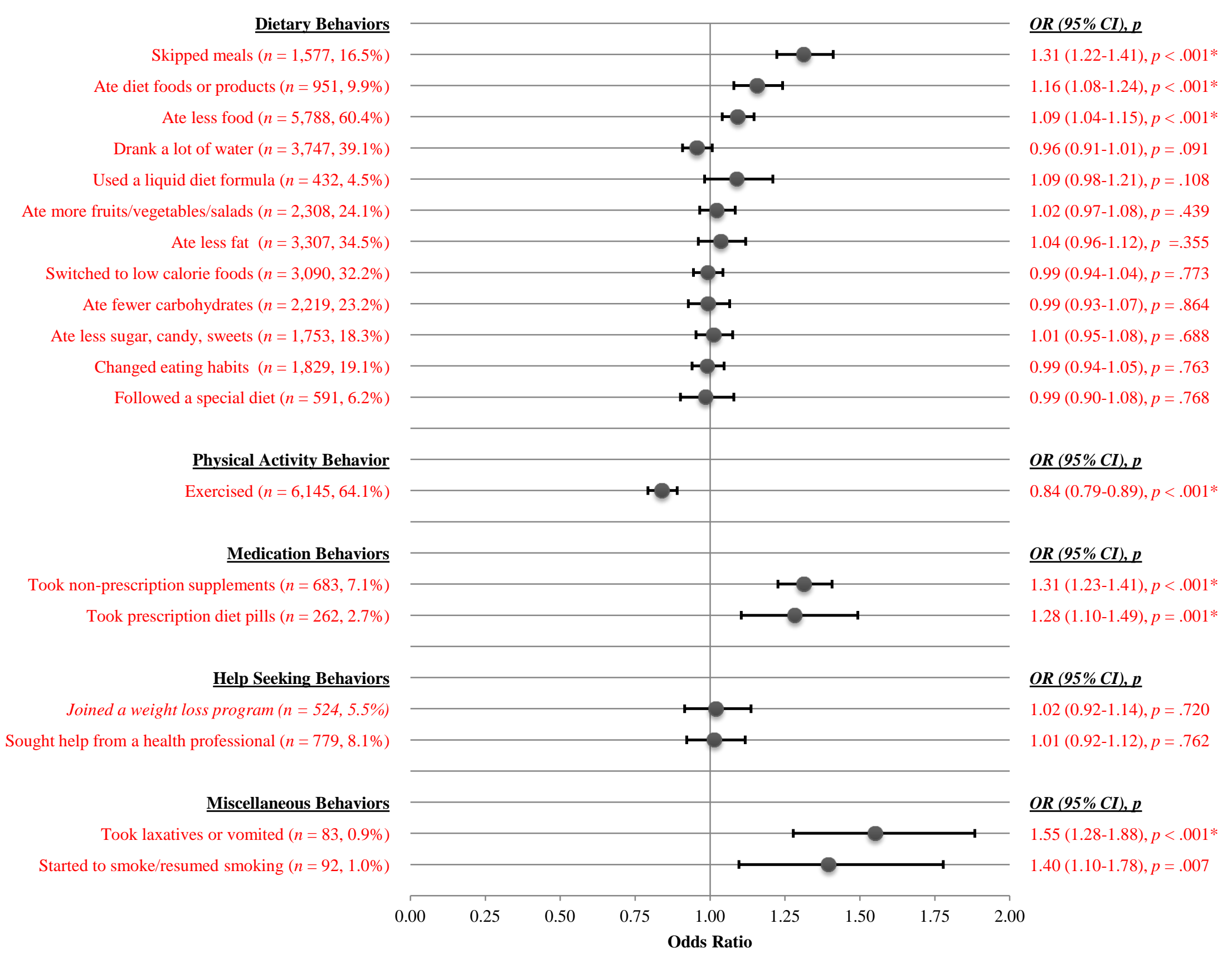




\section{Figure Caption}

Figure 1. Results of logistic regression models examining the relationships between depressive symptom severity (PHQ-9 total, z-scored) and weight loss behaviors. Models were analyzed using Sample \#2 ( $n=9,852$ adults who made a weight loss attempt in the past year) and were adjusted for age, gender, race/ethnicity, education level, body mass index, and NHANES sampling design. PHQ-9=Patient Health Questionnaire-9. OR=odds ratio. $\mathrm{CI}=$ confidence interval.

*Holm-Bonferroni adjusted $p<.05$. 


\section{Highlights}

- Greater depressive symptoms are not associated with odds of trying to lose weight.

- Among those who tried to lose weight:

○ Depressive symptoms related to use of ineffective/unhealthy weight loss behaviors

○ Examples of ineffective behaviors: skipping meals, taking laxatives/vomiting.

○ Depressive symptoms related to avoidance of effective behaviors (exercise).

- Depression may be another mechanism explaining excess risk of obesity in depressed adults.

- Weight loss behaviors may be a modifiable target for future depression interventions. 